\title{
Positive and negative affect of university and college students during COVID-19 outbreak: a network-based survey
}

\author{
Yali Wang ${ }^{1,2,4,5} \cdot$ Xiang Jing ${ }^{1,3,4,5} \cdot$ Wantong Han ${ }^{1,4,5} \cdot$ Yurong Jing ${ }^{1,4,5} \cdot$ Lingzhong $\mathrm{Xu}^{1,4,5}$
}

Received: 14 March 2020/Revised: 5 August 2020 / Accepted: 16 September 2020/Published online: 15 October 2020

(C) Swiss School of Public Health (SSPH+) 2020

\begin{abstract}
Objectives To understand the status of positive affect (PA) and negative affect (NA) on university and college students, and to explore the determinants during the COVID-19 outbreak.

Methods Our data were from network-based survey, and 17,876 participants completed the questionnaire. $t$ test, one-way ANOVA and multiple linear regression model were performed using PANAS (Positive and Negative Affect Schedule) score as the dependent variable.

Results Of 17,876 participants, the mean score of PA was $25.5 \pm 7.3$, while NA was $19.1 \pm 7.1$. Multiple linear regression models showed that there are some common determinants of PA and NA, such as education, health literacy on communicable diseases, satisfaction with measures for epidemic prevention and control, risk of infection, impact of the outbreak on daily life, sleep duration and frequency of hand washing in the past 2 weeks. Besides, whether the student is a medical major and whether outing in the past 2 weeks were specific determinants of PA, and frequency of masks wearing was specific determinant of NA.

Conclusions The outbreak of COVID-19 is detrimental to university and college students' affect. During the outbreak response, we should strengthen the guidance and regulation for negative affect and pay attention to improving the positive affect of university and college students.
\end{abstract}

Keywords COVID-19 - PANAS · University and college students

\section{Abbreviations \\ PANAS The Positive and Negative affect schedule \\ PA Positive affect \\ NA Negative affect}

Xiang Jing co-author.

Lingzhong Xu

lzxu@sdu.edu.cn

Yali Wang

wyl_0719@163.com

Xiang Jing

13209897715@163.com

Wantong Han

hanwant@163.com

Yurong Jing

yrjing7@163.com

\section{Introduction}

COVID-19 is the infectious disease caused by the most recently discovered coronavirus; it was declared a pandemic by WHO and affecting many countries globally (Bruin et al. 2020). As of 24:00 on March 7, 2020, a total of

2 Henan Provincial Center for Disease Control and Prevention, Zhengzhou 450016, Henan, China

3 Yuncheng Central Hospital, Yuncheng 044000, Shanxi, China

4 NHC Key Laboratory of Health Economics and Policy Research, Cheeloo College of Medicine, Shandong University, Jinan 250012, Shandong, China

5 Shandong University, Center for Health Economics Experiment and Public Policy Research, Jinan 250012, Shandong, China

1 School of Public Health, Cheeloo College of Medicine, Shandong University, Jinan 250012, Shandong, China 
80,695 patients with COVID-19 were diagnosed, including 3097 deaths and 57,065 cures in China (China, National Health Commission of the People's Republic of China 2020). Many studies have shown that widespread outbreaks of COVID-19 are associated with psychological distress and caused public panic (Bao et al. 2020; Rajkumar 2020; $\mathrm{Li}$ et al. 2020). In order to control the outbreak of COVID19 , the Chinese government has issued a series of executive orders, such as travel bans, and encouraging people to stay at home for quarantine. And affected by the outbreak of COVID-19, universities and colleges have delayed the return of students to school. As of March 8, more than 20 days have passed since the normal start time, and the date of back to school is unknown. For university and college students, the rapid spread of COVID-19 and the discomfort of being at home for a long time caused that they are vulnerable to mental stress and emotional distress.

Affect is regarded as psychological states that refers to personal evaluative feeling for objective things (DiazGarcia et al. 2020). There were two independent dimensions, including positive affect and negative affect (Garcia 2012). Stable and optimistic affect usually means good mental states. The recent COVID-19 outbreak has been deemed a global health emergency (Sohrabi et al. 2020). When it occurs, individuals will inevitably have negative emotional experience. Negative emotions can lead to a wider range of individual and social behavior problems as well as fear and anxiety, which can be accompanied by acute and chronic stress that may compromise immune function and increase susceptibility to stress-related physiological disorders (Gao 2009). At the same time, we should not ignore the positive emotions. Positive emotional experience can promote the effective emotional control of individuals, experience positive emotions such as gratitude, interest and love; after an emergency, it can help resilient individuals fight depression (Vanderlind et al. 2020). In order to prevent the pandemic from escalating, universities are postponed to start school; moreover, university and college students must reduce their travels, which prevents them from studying and participating in social activities and may affect their learning progress and their mood. The existing researches on psychological distress that caused by COVID-19 mainly focus on anxiety, depression and selfreported stress; the positive affect and negative affect are still unclear. Research has shown that maintaining appropriate levels of both positive and negative emotions under stress can make it easier for individuals to adapt to the environment. University and college students are in the drastic changes period of physical and mental development, and it is also the period in which their outlook on life and world gradually takes shape. Emotions have obvious characteristics of the transition period and are easily excited and unstable. At this time, their understanding of society and self-knowledge is not mature enough, they are poor in adapting to society pressure, they are easily influenced by the external environment and self-contradictions, and they often cannot correctly handle the incidents they encountered. Faced with a major event such as COVID-19, their emotions will inevitably be affected (Zhao et al. 2020). Therefore, it is necessary to pay attention to the positive and negative emotions of university and college students during the pandemic. Based on above, our research hypothesizes that university and college students' positive and negative affect would be influenced in the face of the pandemic and further explored the determinants of positive and negative affect. The aim of this study was to understand the positive and negative affect of university and college students and explore the determinants.

\section{Methods}

\section{Sample}

The survey was conducted on February 20-22, 2020, and network-based survey was used for data collection. In China, higher education is divided into four levels: junior college students (two- and three-year students), undergraduate students, master students and doctoral students. And our subjects were these university and college students from 31 provinces in mainland China. The participants voluntarily completed the questionnaire online based on the reading instructions, with reassurance of no penalty for non-participation. Participants were assured that personal information would not be shared with others without their permission. A total of 18,294 questionnaires were collected, there were 17,876 valid questionnaires after excluding invalid questionnaires, and the effective rate of the questionnaire was $97.7 \%$.

\section{Independent variables and dependent variable}

The questionnaire included the basic information of the sample, the infectious disease prevention part of (China National Health Literacy Monitoring Questionnaire) and the PANAS (Positive and Negative Affect Schedule, PANAS). The basic information included gender (male, female), education (two- and three-year students, undergraduate, master and above), residence (village, town, city), major (medical, non-medical), health literacy on communicable diseases (yes, no), satisfaction with local pandemic prevention and control measures (high, moderate, low), concern about the outbreak (high, moderate, low), self-perceived risk of infection (high, moderate, low), the impact of the outbreak on daily life (high, moderate, low), sleep duration (short sleep, normal sleep, long sleep), 
Table 1 Characteristics of university and college students in network-based survey, China, February 20-22, 2020

\begin{tabular}{|c|c|c|c|c|c|}
\hline Variables & $N(\%)$ & $\mathrm{PA}(\bar{x} \pm s)$ & $t / F$ & $\mathrm{NA}(\bar{x} \pm s)$ & $t / F$ \\
\hline Total & $17,876(100)$ & $25.5 \pm 7.3$ & & $19.1 \pm 7.1$ & \\
\hline Gender & & & 1.184 & & 1.071 \\
\hline Male & $5058(28.3)$ & $25.6 \pm 8.1$ & & $19.2 \pm 7.9$ & \\
\hline Female & $12,818(71.7)$ & $25.5 \pm 7.0$ & & $19.1 \pm 6.8$ & \\
\hline Education & & & $211.890 * *$ & & $24.193 * *$ \\
\hline Two- and three-year students & $9230(51.6)$ & $24.5 \pm 7.6$ & & $18.8 \pm 7.2$ & \\
\hline Undergraduate & $8185(45.8)$ & $26.6 \pm 6.9$ & & $19.5 \pm 7.1$ & \\
\hline Master and above & $461(2.6)$ & $27.6 \pm 6.8$ & & $19.8 \pm 7.0$ & \\
\hline Residence & & & 0.816 & & 2.279 \\
\hline Village & $9113(51.0)$ & $25.6 \pm 7.2$ & & $19.2 \pm 7.0$ & \\
\hline Town & $4524(25.3)$ & $25.4 \pm 7.3$ & & $19.1 \pm 7.0$ & \\
\hline City & 4239 (23.7) & $25.6 \pm 7.6$ & & $19.0 \pm 7.5$ & \\
\hline Major & & & $2.860^{*}$ & & $2.004 *$ \\
\hline Medical & $10,972(61.4)$ & $25.7 \pm 7.0$ & & $19.0 \pm 7.2$ & \\
\hline Non-medical & 6904 (38.6) & $25.4 \pm 7.5$ & & $19.3 \pm 7.1$ & \\
\hline $\begin{array}{l}\text { Health literacy on } \\
\text { communicable diseases }\end{array}$ & & & $-12.621^{* *}$ & & $2.035^{*}$ \\
\hline No & $14,488(81.0)$ & $25.2 \pm 7.4$ & & $19.2 \pm 7.2$ & \\
\hline Yes & $3388(19.0)$ & $26.9 \pm 6.9$ & & $18.9 \pm 6.8$ & \\
\hline $\begin{array}{l}\text { Satisfaction with measures } \\
\text { for pandemic prevention } \\
\text { and control }\end{array}$ & & & 17.936 & & $108.395 * *$ \\
\hline High & $12,960(72.5)$ & $25.7 \pm 7.4$ & & $18.7 \pm 7.0$ & \\
\hline Moderate & $4632(25.9)$ & $25.1 \pm 6.9$ & & $20.2 \pm 7.2$ & \\
\hline Low & $284(1.6)$ & $24.4 \pm 8.6$ & & $22.6 \pm 8.9$ & \\
\hline Concern about the outbreak & & & $309.171 * *$ & & $30.487 * *$ \\
\hline High & $8195(45.8)$ & $26.8 \pm 7.7$ & & $19.5 \pm 7.5$ & \\
\hline Moderate & $9259(51.8)$ & $24.6 \pm 6.7$ & & $18.9 \pm 6.8$ & \\
\hline Low & $422(2.4)$ & $20.7 \pm 7.6$ & & $17.4 \pm 7.5$ & \\
\hline The risk of infection & & & $5.436^{*}$ & & $232.262 * *$ \\
\hline High & $272(1.5)$ & $27.0 \pm 9.1$ & & $23.7 \pm 9.7$ & \\
\hline Moderate & $2398(13.4)$ & $25.6 \pm 6.8$ & & $21.6 \pm 7.3$ & \\
\hline Low & $15,206(85.1)$ & $25.5 \pm 7.4$ & & $18.7 \pm 7.0$ & \\
\hline Impact of the outbreak & & & $95.555^{* *}$ & & $946.300 * *$ \\
\hline High & $6492(36.3)$ & $26.4 \pm 7.2$ & & $21.7 \pm 7.4$ & \\
\hline Moderate & $7294(40.8)$ & $25.3 \pm 6.9$ & & $18.7 \pm 6.6$ & \\
\hline Low & $4090(22.9)$ & $24.5 \pm 8.1$ & & $15.8 \pm 6.1$ & \\
\hline Sleep duration & & & $11.091 * *$ & & $27.382 * *$ \\
\hline Short sleep & $621(3.5)$ & $24.6 \pm 8.4$ & & $21.0 \pm 8.5$ & \\
\hline Normal sleep & $8751(49.0)$ & $25.8 \pm 7.2$ & & $18.9 \pm 7.0$ & \\
\hline Long sleep & 8504 (47.6) & $25.4 \pm 7.4$ & & $19.2 \pm 7.2$ & \\
\hline Outing in the past 2 weeks & & & $5.590 *$ & & 1.256 \\
\hline Stay at home all the time & $13,538(75.7)$ & $25.5 \pm 7.3$ & & $19.1 \pm 7.1$ & \\
\hline Walk around the house & 3781 (21.2) & $25.9 \pm 7.1$ & & $19.2 \pm 7.1$ & \\
\hline Often go out & $72(0.4)$ & $26.3 \pm 9.0$ & & $20.3 \pm 8.9$ & \\
\hline Others & $485(2.7)$ & $24.6 \pm 8.5$ & & $19.3 \pm 8.4$ & \\
\hline Frequency of masks wearing & & & $7.321 *$ & & $11.980 * *$ \\
\hline Every time & $16,098(90.1)$ & $25.6 \pm 7.3$ & & $19.0 \pm 7.1$ & \\
\hline Often & $1059(5.9)$ & $25.5 \pm 7.3$ & & $20.1 \pm 7.4$ & \\
\hline
\end{tabular}


Table 1 (continued)

\begin{tabular}{|c|c|c|c|c|c|}
\hline Variables & $N(\%)$ & $\mathrm{PA}(\bar{x} \pm s)$ & $t / F$ & $\mathrm{NA}(\bar{x} \pm s)$ & $t / F$ \\
\hline Not at all & $719(4.0)$ & $24.5 \pm 7.9$ & & $19.4 \pm 7.7$ & \\
\hline Frequency of hand washing in the past 2 weeks & & & $75.164 * *$ & & $13.440 * *$ \\
\hline Increased significantly & $13,714(76.7)$ & $25.9 \pm 7.3$ & & $19.3 \pm 7.1$ & \\
\hline As usual & $4008(22.4)$ & $24.4 \pm 7.3$ & & $18.6 \pm 7.2$ & \\
\hline Others & $154(0.9)$ & $23.2 \pm 9.3$ & & $18.3 \pm 8.9$ & \\
\hline
\end{tabular}

$* * P<0.001, * P<0.05$, PA and NA were measured by PANAS scale (Watson et al. 1988)

outing in the past 2 weeks (stay at home all the time, walk around the house, often go out, others), frequency of masks wearing (every time, often, not at all) and hand washing in the past 2 weeks (increased significantly, as usual. Others). In our study, positive and negative affect schedule (PANAS) (Watson et al. 1988) was used to assess positive affect (PA) and negative affect (NA). The PANAS includes 20 items, which was divided into two PANAS subscales (PANAS-Positive and PANAS-Negative). And reliability analysis showed that two subscales have good reliability, and the Cronbach alpha is 0.891 and 0.929 , respectively.

\section{Data analysis}

We presented descriptive statistics on the basic information of the sample, the sociodemographic characteristics were presented in percentages, and scores of two subscales were presented in mean $\pm \mathrm{SD}$. $\mathrm{T}$ test and one-way ANOVA were used to compare the scores of PANAS across different groups. And multiple linear regression model was performed to explore the determinants of positive and negative affect. In our study, NA score and PA score were, respectively, set as the dependent variables for further linear regression. IBM SPSS 24.0 was used for data analysis, and $P$-values $<0.05$ were considered to be statistically significant.

\section{Results}

Among the 17,876 participants, 5058 (28.3\%) were males, $12,818(71.7 \%)$ were females. Generally speaking, half of the participants were two- and three-year students $(51.6 \%)$, half of the participants live in village $(51.0 \%)$, majority of the participants were medical professionals $(61.4 \%)$ (Table 1). The mean score of PA was $25.5 \pm 7.3$, while NA was $19.1 \pm 7.1$.

Table 2 showed the determinants of PA and NA among participants. The results indicated that the undergraduate, master and above had higher PA score than the two- and three-year students $(\beta=2.872,2.826$, respectively, both
$P<0.001)$, the undergraduate also had lower NA score than the two- and three-year students $(\beta=0.572$, $P<0.001)$; compared with non-medical students, medical students had higher PA score $(\beta=0.847, P<0.001)$; participants with health literacy had higher PA score $(\beta=1.399, P<0.001)$ and lower NA score $(\beta=-0.311$, $P<0.05)$; the participants who feel moderate or dissatisfied with measures for pandemic prevention and control had lower PA score $(\beta=-0.421,-1.334$, respectively, both $P<0.05)$ and higher NA score than those satisfied ( $\beta=1.072,2.661$, respectively, both $P<0.001$ ); the lower the level of concern for the outbreak, the lower the scores of PA $(\beta=-2.037, \quad-5.518, \quad$ respectively, both $P<0.001)$ and NA $(\beta=-0.413,-1.598$, respectively, both $P<0.001)$; those with a moderate and low risk of infection had lower PA $(\beta=-1.030,-0.914$, respectively, both $P<0.05)$ and NA scores than those with a high risk of infection $(\beta=-1.326,-3.199$, respectively, both $P<0.05)$; the lower the impact of the outbreak, the lower the scores of PA $(\beta=-0.688,-1.414$, respectively, both $P<0.001)$ and NA $(\beta=-2.696,-5.195$, respectively, both $P<0.001)$; participants with normal and long sleep had higher PA score $(\beta=0.838,0.633$, respectively, both $P<0.05)$ and lower NA score $(\beta=-1.513, \quad-1.171$, respectively, both $P<0.001)$; compare with stay at home all the time, the PA score of who walk around the house was higher $(\beta=0.484$, $P<0.001)$; the lower the frequency of wearing a mask, the lower the NA score $(\beta=0.983, P<0.001)$; the less frequent hand washing over the past 2 weeks, the lower the PA score $(\beta=-1.192, \quad-1.484$, respectively, both $P<0.05)$, participants who washed their hands as often as usual had lower NA score than those who washed their hands significantly more $(\beta=-0.535, P<0.001)$.

\section{Discussion}

In our study, during COVID-19 outbreak, university and college students' positive affect was at a moderate level, while negative affect was moderately low, which were 
Table 2 Determinants associated with positive and negative affects among university and college students in network-based survey, China, February 20-22, 2020

\begin{tabular}{|c|c|c|c|c|c|c|}
\hline \multirow[t]{2}{*}{ Variables } & \multicolumn{3}{|l|}{ PA } & \multicolumn{3}{|l|}{ NA } \\
\hline & $\beta$ & SE & $95 \% \mathrm{CI}$ & $\beta$ & SE & $95 \% \mathrm{CI}$ \\
\hline \multicolumn{7}{|c|}{ Education (ref: two- and three-year students) } \\
\hline Undergraduate & $2.872 * *$ & 0.127 & $(2.623,3.122)$ & $0.572 * *$ & 0.122 & $(0.334,0.811)$ \\
\hline Master and above & $2.826 * *$ & 0.338 & $(2.163,3.489)$ & 0.366 & 0.323 & $(-0.268,1.000)$ \\
\hline \multicolumn{7}{|l|}{ Major (ref: non-medical) } \\
\hline Medical & $0.847 * *$ & 0.128 & $(0.596,1.098)$ & 0.151 & 0.122 & $(-0.089,0.391)$ \\
\hline \multicolumn{7}{|c|}{$\begin{array}{l}\text { Health literacy on communicable diseases } \\
\text { (ref: no) }\end{array}$} \\
\hline Yes & $1.399 * *$ & 0.136 & $(1.132,1.665)$ & $-0.311 *$ & 0.130 & $(-0.566,-0.057)$ \\
\hline \multicolumn{7}{|c|}{$\begin{array}{l}\text { Satisfaction with measures for pandemic } \\
\text { prevention and control (ref: high) }\end{array}$} \\
\hline Moderate & $-0.421 *$ & 0.125 & $(-0.666,-0.176)$ & $1.072 * *$ & 0.120 & $(0.837,1.306)$ \\
\hline Low & $-1.334 *$ & 0.425 & $(-2.166,-0.501)$ & $2.661 * *$ & 0.406 & $(1.866,3.456)$ \\
\hline \multicolumn{7}{|c|}{ Concern about the outbreak (ref: high) } \\
\hline Moderate & $-2.037 * *$ & 0.111 & $(-2.255,-1.819)$ & $-0.413 * *$ & 0.106 & $(-0.621,-0.205)$ \\
\hline Low & $-5.158 * *$ & 0.359 & $(-5.863,-4.454)$ & $-1.598 * *$ & 0.343 & $(-2.271,-0.925)$ \\
\hline \multicolumn{7}{|c|}{ The risk of infection (ref: high) } \\
\hline Moderate & $-1.030 *$ & 0.451 & $(-1.915,-0.145)$ & $-1.326^{*}$ & 0.431 & $(-2.172,-0.481)$ \\
\hline Low & $-0.914 *$ & 0.433 & $(-1.763,-0.066)$ & $-3.199 * *$ & 0.414 & $(-4.01,-2.388)$ \\
\hline \multicolumn{7}{|c|}{ Impact of the outbreak (ref: high) } \\
\hline Moderate & $-0.688 * *$ & 0.122 & $(-0.927,-0.449)$ & $-2.696 * *$ & 0.116 & $(-2.924,-2.467)$ \\
\hline Low & $-1.414 * *$ & 0.144 & $(-1.697,-1.131)$ & $-5.195 * *$ & 0.138 & $(-5.465,-4.924)$ \\
\hline \multicolumn{7}{|c|}{ Sleep duration (ref: short sleep) } \\
\hline Normal sleep & $0.838^{*}$ & 0.293 & $(0.263,1.412)$ & $-1.513 * *$ & 0.280 & $(-2.062,-0.964)$ \\
\hline Long sleep & $0.633^{*}$ & 0.293 & $(0.059,1.208)$ & $-1.171 * *$ & 0.280 & $(-1.720,-0.621)$ \\
\hline \multicolumn{7}{|c|}{$\begin{array}{l}\text { Outing in the past } 2 \text { weeks (ref: stay at home } \\
\text { all the time) }\end{array}$} \\
\hline Walk around the house & $0.484 * *$ & 0.130 & $(0.228,0.740)$ & & & \\
\hline Often go out & 1.115 & 0.832 & $(-0.515,2.746)$ & & & \\
\hline Others & -0.257 & 0.328 & $(-0.900,0.387)$ & & & \\
\hline \multicolumn{7}{|c|}{ Frequency of masks wearing (ref: every time) } \\
\hline Often & 0.367 & 0.225 & $(-0.073,0.807)$ & $0.983 * *$ & 0.214 & $(0.564,1.402)$ \\
\hline Not at all & -0.153 & 0.274 & $(-0.691,0.385)$ & 0.509 & 0.262 & $(-0.004,1.023)$ \\
\hline \multicolumn{7}{|c|}{$\begin{array}{l}\text { Frequency of hand washing in the past } 2 \\
\text { weeks (ref: increased significantly) }\end{array}$} \\
\hline As usual & $-1.192 * *$ & 0.131 & $(-1.449,-0.934)$ & $-0.535^{* *}$ & 0.126 & $(-0.782,-0.289)$ \\
\hline Others & $-1.484 *$ & 0.577 & $(-2.616,-0.353)$ & -0.776 & 0.547 & $(-1.848,0.296)$ \\
\hline
\end{tabular}

$* * P<0.001, * P<0.05, \mathrm{PA}$ and NA were measured by PANAS scale (Watson et al. 1988)

lower than the research results of university and college students in general (Lin 2018). It can be seen that under the stress of the pandemic, the emotions of university and college students have been affected.

The research results showed that participants with high academic qualifications, medical majors and health literacy had higher PA scores; this group of people had a certain amount of knowledge, a comprehensive understanding of the pandemic situation and had taken in place preventive measures, and medical students have rich medical knowledge, so this group is more active when facing the pandemic situation (Sun 2019). In addition, the undergraduates had higher NA scores than the two- and three-year students, which also reflects the independence between 
positive and negative affects, that is, when an individual has a positive emotional experience, it does not mean that there is no negative emotional experience (Huang et al. 2003). As an important force in social development, undergraduates are willing to accept new things and are highly active on social media, but their experience is still shallow and they are prone to generate intense emotions faced with major events.

Our study found that individuals who were lower satisfaction with control measures were associated with lower PA and higher NA. When individuals deemed that measures for pandemic were difficult to achieve the purpose of prevention and control, their negative emotional experience will be more intense. This finding showed that it is necessary to improve public's satisfaction about measures for pandemic prevention and control. Besides, concern about the outbreak, the risk of infection and impact of the outbreak also exerted statistically significant effect on PA and NA. These factors generally reflected individual psychological perception of the outbreak of COVID-19. Individuals who had lower perception for the outbreak of COVID-19 had lower PA and NA, which means individual affect was in a stable state correspondingly.

Finally, we found that health behaviors had significant effects on PA and NA, including sleep duration, outing in the past 2 weeks, frequency of masks wearing and hand washing. Among them, sleep duration was positively correlated with PA, while were negatively with NA. This might be explained by the fact that sufficient sleep can recognize and control negative thoughts, while lack of sleep may lead to greater mental stress. Previous studies have proved that insufficient sleep was associated with depression (Williams et al. 2020). And compared with university and college students who has been staying at home all the time, students who walk around the house had higher PA. Without a doubt, going out is a good way to relax and relieve stress. However, considering the severity of the pandemic, people should stay at home and seek other ways to alleviate mental stress. In addition, compared with those who wear masks every time, those who often wear masks had higher NA scores. Individuals who often wear masks means had recognized the severity of COVID-19; however, they failed to wear masks every time due to other factors (such as cannot buy masks needed). This may be the reason why their negative effect was higher. Similarly, washing hands frequently was considered as an effective personal protection measure. Obviously, individuals who washed hands as often as usual have a low degree of concern about the outbreak of COVID-19. Correspondingly, their positive affect and negative affect were more stable.

There are some limitations in our study. Firstly, our data are from cross-sectional survey, and we did not examine the causal relationship among different variables. Secondly, our data were entirely based on self-reporting. This may have affected the veracity of these information to some extents. Finally, there may be more confounding factors than those available for consideration in this study.

During the outbreak response, we should strengthen the guidance and regulation of negative emotions for university and college students, and at the same time, we must pay attention to improving the positive emotions of students. University and college students should strengthen their confidence, properly control their attention to the pandemic situation of COVID-19, try to pay attention to official information, adhere to healthy behaviors such as washing hands and wearing a mask and arrange schedules. Relevant government departments and universities should use social platforms to popularize COVID-19-related health knowledge for students, help university and college students to understand COVID-19 correctly and comprehensively, and at the same time, mental counseling should be carried out for university and college students to protect their emotional health.

Acknowledgements We thank the Ms L Lu, Ms Y Li, Ms Y Xia and Mr A Jiao who collected these data. We are also grateful to also thank Ms J Zhang and Ms Z Qin for technical guidance. The authors thank all the participants for their cooperation.

Funding This research was funded by the National Natural Science Foundation of China (Grant Number 71974118).

\section{Compliance with ethical standards}

Conflict of interest The authors declare that they have no conflict of interest.

Informed consent Informed consent was obtained from all individual participants included in the study.

\section{References}

Bao YP, Sun YK, Meng SQ, Shi J, Lu L (2020) 2019-nCoV epidemic: address mental health care to empower society. Lancet 395:E37E38. https://doi.org/10.1016/S0140-6736(20)30309-3

Bruin YBD, Lequarre AS, McCourt J et al (2020) Initial impacts of global risk mitigation measures taken during the combatting of the COVID-19 pandemic. Saf Sci 128:104773-104780. https:// doi.org/10.1016/j.ssci.2020.104773

China, National Health Commission of the People's Republic of China (2020) Update on COVID-19 as of 24:00, 7 March 2020. National Health Commission of the People's Republic of China. http://www.nhc.gov.cn/xcs/yqtb/202003/b4c328ff60874b99ba6 ce8caf827987b.shtml. Accessed 8 Mar 2020

Diaz-Garcia A, Gonzalez-Robles A, Mor S, Mira A et al (2020) Positive and Negative Affect Schedule (PANAS): psychometric properties of the online Spanish version in a clinical sample with emotional disorders. BMC Psychiatry 20:56. https://doi.org/10. $1186 / \mathrm{s} 12888-020-2472-1$ 
Gao J (2009) A study on physiological responding and subjective experiences of emotion regulation on positive and negative emotion. Dissertation, Liaoning Normal University

Garcia D (2012) The affective temperaments: differences between adolescents in the big five model and Cloninger's psychobiological model of personality. J Happiness Stud 13:999-1017. https://doi.org/10.1007/s10902-011-9303-5

Huang L, Yang T, Ji Z (2003) Applicability of the Positive and Negative Affect Scale in Chinese. Chin Mental Health J 17:54-56

Li Z, Ge J, Yang M et al (2020) Vicarious traumatization in the general public, members, and non-members of medical teams aiding in COVID-19 control. Brain Behav Immun. https://doi. org/10.1016/j.bbi.2020.03.007

Lin D (2018) Research on the effect of resilience intervention of college students. Dissertation, Northeast Normal University

Rajkumar RP (2020) COVID-19 and mental health: a review of the existing literature. Asian J Psychiatry 52:102066-102070. https://doi.org/10.1016/j.ajp.2020.102066

Sohrabi C, Alsafi Z, O'Neill N et al (2020) World Health Organization declares Global Emergency: a review of the 2019 Novel Coronavirus (COVID-19). Int J Surg 76:71-76. https://doi.org/ 10.1016/j.ijsu.2020.02.034
Sun Z (2019) Application of positive psychology in college students' mental health education. J Zaozhuang Univ 36:131-135

Vanderlind WM, Millgram Y, Baskin-Sommers AR, Clark MS, Joormann J (2020) Understanding positive emotion deficits in depression: from emotion preferences to emotion regulation. Clin Psychol Rev 76:101826. https://doi.org/10.1016/j.cpr.2020. 101826

Watson D, Clark LA, Tellegen A (1988) Development and validation of brief measures of positive and negative affect: the PANAS scales. J Pers Soc Psychol 54:1063-1070. https://doi.org/10. 1037/0022-3514.54.6.1063

Williams AB, Dzierzewski JM, Griffin SC, Lind MJ, Dick D, Rybarczyk BD (2020) Insomnia disorder and behaviorally induced insufficient sleep syndrome: prevalence and relationship to depression in college students. Behav Sleep Med 18:275-286. https://doi.org/10.1080/15402002.2019.1578772

Zhao B, Cai TJ, Zhang ZH (2020) Presentation and adjustment of college students' emotional state under COVID-19 epidemicbased on the perspective of self-care. China Youth Study. https:// doi.org/10.19633/j.cnki.11-2579/d.2020.0053

Publisher's Note Springer Nature remains neutral with regard to jurisdictional claims in published maps and institutional affiliations. 